\title{
1 Worldwide tracing of mutations and the evolutionary dynamics of
}

\section{SARS-CoV-2}

3 Zhong-Yin Zhou ${ }^{1, \dagger}$, Hang Liu ${ }^{1,2, \dagger}$, Yue-Dong Zhang ${ }^{1,2}$, Yin-Qiao Wu ${ }^{1,2}$, Min-Sheng

4 Peng ${ }^{1}$, Aimin $\mathrm{Li}^{3}$, David M. Irwin ${ }^{4}$, Haipeng $\mathrm{Li}^{5,6}$, Jian $\mathrm{Lu}^{7}$, Yiming Bao ${ }^{8}$, Xuemei

$5 \quad \mathrm{Lu}^{1,6}$, Di Liu ${ }^{9}$, and Ya-Ping Zhang ${ }^{1,6, *}$

6

$7 \quad{ }^{1}$ State Key Laboratory of Genetic Resources and Evolution, Kunming Institute of

8 Zoology, Chinese Academy of Sciences, Kunming, Yunnan, 650223, China.

$9{ }^{2}$ Kunming College of Life Science, University of Chinese Academy of Sciences,

10 Kunming, Yunnan, 650204, China.

$11{ }^{3}$ Shaanxi Key Laboratory for Network Computing and Security Technology, School

12 of Computer Science and Engineering, Xi'an University of Technology, Xi'an,

13 Shaanxi, 710048, China.

$14{ }^{4}$ Department of Laboratory Medicine and Pathobiology, University of Toronto,

15 Toronto, Canada.

$16{ }^{5}$ CAS Key Laboratory of Computational Biology, CAS-MPG Partner Institute for

17 Computational Biology, Shanghai Institute of Nutrition and Health, University of

18 Chinese Academy of Sciences, Chinese Academy of Sciences, China

$19{ }^{6}$ Center for Excellence in Animal Evolution and Genetics, Chinese Academy of

20 Sciences, Kunming, 650223, China.

$21{ }^{7}$ State Key Laboratory of Protein and Plant Gene Research, Center for Bioinformatics,

22 School of Life Sciences, Peking University, Beijing 100871, China; 
$23{ }^{8}$ National Genomics Data Center, Beijing Institute of Genomics, Chinese Academy

24 of Sciences and China National Center for Bioinformation, Beijing 100101, China

$25{ }^{9}$ CAS Key Laboratory of Special Pathogens, Wuhan Institute of Virology, Center for

26 Biosafety Mega-Science, Chinese Academy of Sciences, Wuhan, China

$27 \dagger$ These authors contributed equally to this work.

28 *Email: zhangyp@mail.kiz.ac.cn. 
Abstract

Understanding the mutational and evolutionary dynamics of SARS-CoV-2 is essential for treating COVID-19 and the development of a vaccine. Here, we analyzed publicly available 15,818 assembled SARS-CoV-2 genome sequences, along with 2,350 raw sequence datasets sampled worldwide. We investigated the distribution of inter-host single nucleotide polymorphisms (inter-host SNPs) and intra-host single nucleotide variations (iSNVs). Mutations have been observed at $35.6 \%(10,649 / 29,903)$ of the bases in the genome. The substitution rate in some protein coding regions is higher than the average in SARS-CoV-2 viruses, and the high substitution rate in some regions might be driven to escape immune recognition by diversifying selection. Both recurrent mutations and human-tohuman transmission are mechanisms that generate fitness advantageous mutations. Furthermore, the frequency of three mutations (S protein, F400L; ORF3a protein, T164I; and ORF1a protein, Q6383H) has gradual increased over advantageous mutations. Our study provides theoretical support for vaccine development and the optimization of treatment for COVID-19. We call researchers to submit raw sequence data to public databases. (COVID-19). This disease has spread worldwide, and as of June 26, 2020, has infected more than nine million humans ${ }^{1}$. The whole-genome sequence of SARSsimilar to human SARS-CoV². Recent studies have indicated that SARS-CoV-2 is more easily transmitted from person to person than SARS-CoV ${ }^{3,4}$. SARS-CoV-2 has

54 also gradually accumulated new mutations that may make it more suitable to the 55 human host ${ }^{5,6}$. 
With the development of next-generation sequencing, it has become possible to

57 conduct large-scale studies to detect inter-host single nucleotide polymorphisms

58 (inter-host SNPs) and intra-host single nucleotide variations (iSNVs) for infectious diseases, such as Ebola virus that can uncover essential information concerning their transmission and evolution ${ }^{7,8}$. Thus, tracing inter-host SNPs and iSNVs, and revealing the evolutionary dynamics of SARS-CoV-2 worldwide is a priority. differences in SNPs from assembled genome sequences and publicly available raw reads. We collected 15,818 high-quality SARS-CoV-2 genome sequences from the publicly accessible GISAID, CNGBdb, GenBank, $\mathrm{GWH}^{9}$ and NMDC databases on May 27, 2020. All of these sequences were aligned against the reference genome (Wuhan-Hu-1, GenBank NC_045512.2) ${ }^{10}$ using MUSCLE ${ }^{11}$, revealing 7,700 interhost SNP sites (4,672 nonsynonymous, 2,570 synonymous, 98 stop gain, 7 stop loss, and 433 noncoding) (Extended Data Table 1). Publicly available raw reads were extracted from 1,364 clinical SARS-CoV-2 samples from Australia, 109 samples from

71 India, 400 samples from the United Kingdom, 112 samples from China ${ }^{12}$, and 365 samples from the United States that were collected between January and May 2020 nonsynonymous, 1,572 synonymous, 261 stop gain, 4 stop loss and 134 noncoding) analyzed, the number of iSNV is much underestimated. 
We then investigated the distribution of inter-host SNPs and iSNVs in each open reading frame (ORF) and found similar trends in the SNP numbers and SNP numbers normalized by the length between inter-host SNPs and iSNVs (Fig. 1, a-d). We then focused on the S-protein receptor binding domain (RBD) as it is being used for monoclonal antibody isolation and vaccine design ${ }^{13,14}$. Several mutations were found in this region, including 68 inter-host nonsynonymous and 65 intra-host nonsynonymous mutations (Fig. 1, a and c). An excess of C-to-T, A-to-G and T-to-C mutations were observed in both the inter-host SNPs and iSNVs, which may be the result of the RNA editing signature of APOBEC deaminases and ADAR enzymes (Fig. 1e). Furthermore, we analyzed the distributions of the numbers of nonsynonymous and synonymous mutations (Fig. 1f). Some regions of the SARSCoV-2 genome have greater numbers of nonsynonymous mutations than the average level (Fig. 1f). The high numbers of nonsynonymous mutations found at the ends of the SARS-CoV-2 genome might stem from errors of genome assembly. The higher numbers of nonsynonymous mutations for some regions might be due to higher substitution rates and increased recombination. To address this, we estimated the substitution rates using BEAST ${ }^{15}$ a set of assembled genomes obtained from patients from Shanghai. A substitution rate of $1.20 \times 10^{-3}$ mutations per site per year $(95 \%$ highest posterior density interval, $6.14 \times 10^{-4}$ to $1.87 \times 10^{-3}$ mutations per site per year) for these SARS-CoV-2 genomes was estimated (Extended Data Table 4), which is similar to the previous estimate of $9.7 \times 10^{-4}$ mutations per site per year ${ }^{16}$. However, higher substitution rates were found for some protein-coding regions (such as genome positions $21,563-22,700$ and $24,300-25,384$ in the S protein), of approximately $2.95 \times 10^{-3}$ to $3.94 \times 10^{-3}$ mutations per site per year (Fig. 1f and Extended Data Table 4).

When the sequences were tested for evidence of recombination using the Recombination Detection Program (RDP $)^{17}$, none was detected in the genomes from 
107 the Shanghai patients. These results suggest that some regions of the SARS-CoV-2

108 genome are experiencing faster rates of evolution.

110 first calculated $\pi$, the nucleotide diversity of the viral populations using pairwise

111 comparisons for each gene from the inter-host sequences. We can evaluate the level of natural selection acting upon the sequences by comparing the frequencies of the synonymous $(\pi \mathrm{S})$ and nonsynonymous $(\pi \mathrm{N})$ polymorphisms ${ }^{18} . \pi \mathrm{S}>\pi \mathrm{N}$ is indicative of

114 purifying selection removing deleterious mutations, $\pi \mathrm{S}<\pi \mathrm{N}$ indicates diversifying

115 selection, and $\pi \mathrm{S}=\pi \mathrm{N}$ suggests neutrality. For most genes, the inter-host sequence data 116 shows that $\pi \mathrm{N}$ is less than $\pi \mathrm{S}$, implying that purifying selection plays a central role in 117 the evolution of these SARS-CoV-2 genes (Extended Data Table 5). For ORF3a and $118 \mathrm{ORF} 8, \pi \mathrm{N}$ was greater than $\pi \mathrm{S}$, although the standard error of the $\pi \mathrm{N}$ values is high

119 (Extended Data Table 5), which prevents us from concluding that diversifying 120 selection is occurring in those two genes. Previous studies have indicated that 121 evolution of the mucin-like domain of the Ebola virus glycoprotein might have been driven by diversifying selection imposed by antibodies ${ }^{7}$. To further investigate regions of the SARS-CoV-2 genome whose evolution might have been driven by

124 diversifying selection, we calculated $\pi \mathrm{N}$ and $\pi \mathrm{S}$ using a sliding window of 30 codons 125 and a step size of 3 codons $^{18}$. Using this sliding window analysis, we found signals for 126 diversifying selection in small regions of the ORF1ab, S, ORF3a, and ORF8 genes 127 (Fig. 2a). $\pi \mathrm{N}$ is greater than $\pi \mathrm{S}$ in one region (Genome position: 23,318-23,488, $\mathrm{S}$ 128 protein: 584-641) in the SD2 domain of the S protein (27 nonsynonymous and 16 129 synonymous within this region) (Fig. 2a). This region, including the fitness advantage 130 pandemic mutation $\mathrm{D}_{6} 14 \mathrm{G}^{5}$, is in the surface-exposed viral protein of the SARS-CoV 131 virus and might be a primary target for antibodies. Thus, antibodies might be the 132 driving force for the diversifying selection seen in this region. Further studies are 
133 needed to test the enrichment of mutations within the B cell epitopes within this 134 region.

The frequency of iSNVs can also be used to reveal selection signals ${ }^{8,19}$. We analyzed the mutated allele frequency (MuAF) using the Wuhan-Hu-1 genome as a

137 reference. We performed the MuAF analysis using a sliding window of 30 codons and 138 a step size of 3 codons. A small region (Genome position: 23,066-23,191, S protein:

139 502-543) of the RBD and SD1 domains of the S protein has a nonsynonymous

140 frequency that is significantly higher than the synonymous frequency (there are 13 141 nonsynonymous and 7 synonymous mutations in this region) (Kolmogorov-Smirnov 142 test, $\mathrm{P}<0.05$ ) (Fig. 2b). Another region (Genome position: 24,218-24,316, S protein: 143 886-918) in the S protein CR and HR1 domains also has a significantly higher 144 nonsynonymous frequency than the synonymous frequency (there are 9 145 nonsynonymous and 4 synonymous mutations in this region) (Fig. 2b) and is 146 overlapping with substitution mutation rate region (block 5 in Extended Data Table 4; 147 genome position: 24,300-27,800). Diversifying selection on these two regions might 148 also be driven by an intra-host escape from antibody recognition.

To study the mechanisms for the generation and transmission of fitness

150 advantage mutations, we first investigated the number of iSNVs over time and noticed 151 that the numbers of iSNVs was generally steady with few fluctuations (Fig. 3a). Thus, 152 we concluded that there was no increase in diversity within intra-host genomes over 153 time. iSNVs provide valuable information about human-to-human transmission and 154 are useful for characterizing human-to-human transmission chains. In the 2,187

155 worldwide cases, which spanned 5 months of the COVID-19 epidemic, 1,988 samples 156 shared at least one iSNVs (Fig. 3b). The shared iSNVs can be explained by 157 superinfection, recurring mutation or human-to-human transmission ${ }^{7}$. We can rule out 158 superinfection, as 2,949 shared iSNVs were detected only in raw reads and were not 
159 identified in the assembled sequences. Additionally, the fitness advantage mutation (G 160 to A change) at position 23,403 (spike protein, D614G, Wuhan-Hu-1, GenBank

161 NC_045512.2) $)^{5}$ is at an intermediate frequency ( 60\% in four samples) in the 162 Australia population, and belong to G clade (Extended Data Fig.2). If superinfection 163 were common in COVID-19 patients, we would expect to see near equal distributions 164 of iSNVs containing individuals in both the A and G clades. From the above results, 165 we conclude that superinfection is not an important reason for the existence of iSNVs.

166 Another possible reason for shared iSNVs is recurring mutation and human-to-human 167 transmission. We can see the shared iSNVs at low frequencies in different countries 168 or regions and at different times. Thus, we cannot exclude the influence of recurring 169 mutation. Importantly, all of the shared iSNVs are unlikely to be the result of only 170 recurring mutations as 23,403 variants were found at $>90 \%$ frequency in 34 different 171 samples from the United Kingdom, the United States, and Australia. The high 172 frequency of the shared iSNVs might arise from human-to-human transmission and/or 173 adaption. In summary, we can conclude that recurrent mutations and human-to174 human transmission could lead to the observed iSNV pattern seen in COVID-19 175 patients.

177 increase in frequency faster than alleles without a fitness advantage. Thus, if a

178 mutation has a selective advantage, then the frequency of this mutation will gradually

179 increase over time in a transmission chain (Fig. 4a). To detect possible fitness 180 advantage mutations in SARS-CoV-2, we analyzed 343 iSNVs in genomes from 181 Shanghai, China, requiring that the iSNVs existed for at least three days and from two 182 samples for each day. Furthermore, we constructed a median-joining haplotype 183 network (Fig. 4b) using PopART (http://popart.otago.ac.nz) and a phylogenetic tree 184 (Extended Data Fig.3) using IQ-TREE for the Shanghai samples ${ }^{20}$. We then calculated the allele frequency and plotted the population-level frequency dynamic 
186 with isolate sampling times for the above iSNVs for each lineage. We found two

187 shared iSNVs (mutation type) in the red cluster and two shared iSNVs in the black

188 cluster that had gradual frequency increases over time (Fig. 4c and Extended Data

189 Fig.4a). The $\mathrm{T}$ to $\mathrm{C}$ mutation (position $22,760, \mathrm{~S}$ protein) causing the F400L amino

190 acid substitution might be a fitness advantage mutation for SARS-CoV-2 in these

191 patients, while another synonymous mutation ( $\mathrm{T}$ to $\mathrm{C}$, position 22,762 ) in the same

192 codon is likely hitchhiking. Another pair of mutations ( $\mathrm{C}$ to T, position 25,883,

193 ORF3a protein, T164I and A to T, position 19413, ORF1a protein, Q6383H) might

194 also provide a fitness advantage within these SARS-CoV-2 patients. Genetic drift

195 could also lead to increases in iSNV frequency. Whereas genetic drift may affect the

196 whole genome, some iSNVs may not follow similar patterns and thus, likely were

197 driven by purifying or neutral selection (Fig. 4d and Extended Data Fig.4b). However,

198 we cannot completely rule out the influence of genetic drift. These mutations, to date,

199 are not yet highly prevalent in COVID-19 patients. We urged researchers to focus on

200 these types of mutations and upload raw data of SARS-CoV-2 genomes to public

201 databases for the monitoring of iSNV frequencies.

In summary, we found that to May 27 , at least $35.6 \%$ of the bases of the SARS-

203 CoV-2 genome harbor one or more mutation and that evolution of mutations in the

204 spike protein gene might have been driven by diversifying selection to increase

205 diversity to escape immune system recognition. Furthermore, we found that recurring

206 mutations and human-to-human transmission are the reasons for the prevalence of the

207 fitness advantage mutations in SARS-CoV-2. Through an analysis of shared iSNVs in

208 SARS-CoV-2 genomes from patients from Shanghai, we found three alleles that

209 might be fitness advantage mutations for SARS-CoV-2. This study provides

210 theoretical support for vaccine development and optimizing treatment. We also

211 strongly recommend that researchers submit raw sequence data and epidemiological 
bioRxiv preprint doi: https://doi.org/10.1101/2020.08.07.242263; this version posted August 10, 2020. The copyright holder for this preprint (which was not certified by peer review) is the author/funder. All rights reserved. No reuse allowed without permission.

212 information of SARS-CoV-2 isolates to public databases, which will improve the

213 detection of fitness advantage mutations of SARS-CoV-2.

\section{Main References}

1 Organization, W. H. WHO Coronavirus disease(COVID-19) Situation Report, https://www.who.int/emergencies/diseases/novel-coronavirus-2019/situationreports/ (2020).

2 Zhou, P. et al. A pneumonia outbreak associated with a new coronavirus of probable bat origin. Nature 579, 270-273, https://doi.org/10.1038/s41586-0202012-7 (2020).

3 Chan, J. F. et al. A familial cluster of pneumonia associated with the 2019 novel coronavirus indicating person-to-person transmission: a study of a family cluster. Lancet (London, England) 395, 514-523, https://doi.org/10.1016/s01406736(20)30154-9 (2020).

4 Wrapp, D. et al. Cryo-EM structure of the 2019-nCoV spike in the prefusion conformation. Science (New York, N.Y.) 367, 1260-1263, https://doi.org/10.1126/science.abb2507 (2020).

5 Korber, B. et al. Tracking changes in SARS-CoV-2 Spike: evidence that D614G increases infectivity of the COVID-19 virus. Cell (2020).

6 Tang, X. et al. On the origin and continuing evolution of SARS-CoV-2. National science review (2020).

7 Park, D. J. et al. Ebola Virus Epidemiology, Transmission, and Evolution during Seven Months in Sierra Leone. Cell 161, 1516-1526, https://doi.org/10.1016/j.cell.2015.06.007 (2015).

$8 \mathrm{Ni}, \mathrm{M}$. et al. Intra-host dynamics of Ebola virus during 2014. Nature microbiology 1, 16151, https://doi.org/10.1038/nmicrobiol.2016.151 (2016).

9 Zhao, W. M. et al. The 2019 novel coronavirus resource. Yi chuan = Hereditas 42, 212-221, https://doi.org/10.16288/j.yczz.20-030 (2020).

$10 \mathrm{Wu}, \mathrm{F}$. et al. A new coronavirus associated with human respiratory disease in China. Nature 579, 265-269, https://doi.org/10.1038/s41586-020-2008-3 (2020).

11 Edgar, R. C. MUSCLE: multiple sequence alignment with high accuracy and high throughput. Nucleic acids research 32, 1792-1797, https://doi.org/10.1093/nar/gkh340 (2004).

12 Zhang, X. et al. Viral and host factors related to the clinical outcome of COVID19. Nature, https://doi.org/10.1038/s41586-020-2355-0 (2020).

$13 \mathrm{Wu}, \mathrm{Y}$. et al. A noncompeting pair of human neutralizing antibodies block COVID-19 virus binding to its receptor ACE2. Science (New York, N.Y.) 368, 1274-1278, https://doi.org/10.1126/science.abc2241 (2020).

14 Dai, L. et al. A Universal Design of Betacoronavirus Vaccines against COVID19, MERS, and SARS. Cell, https://doi.org/10.1016/j.cell.2020.06.035 (2020).

15 Suchard, M. A. et al. Bayesian phylogenetic and phylodynamic data integration using BEAST 1.10. Virus evolution 4, vey016, https://doi.org/10.1093/ve/vey016 (2018). 
16 van Dorp, L. et al. No evidence for increased transmissibility from recurrent mutations in SARS-CoV-2. bioRxiv, https://doi.org/doi.org/10.1101/2020.05.21.108506 (2020).

17 Martin, D. P., Murrell, B., Golden, M., Khoosal, A. \& Muhire, B. RDP4: Detection and analysis of recombination patterns in virus genomes. Virus evolution 1, vev003, https://doi.org/10.1093/ve/vev003 (2015).

18 Nelson, C. W., Moncla, L. H. \& Hughes, A. L. SNPGenie: estimating evolutionary parameters to detect natural selection using pooled next-generation sequencing data. Bioinformatics (Oxford, England) 31, 3709-3711, https://doi.org/10.1093/bioinformatics/btv449 (2015).

$19 \mathrm{Li}$, Y. et al. Resequencing of 200 human exomes identifies an excess of lowfrequency non-synonymous coding variants. Nature genetics 42, 969-972, https://doi.org/10.1038/ng.680 (2010).

20 Minh, B. Q. et al. IQ-TREE 2: New Models and Efficient Methods for Phylogenetic Inference in the Genomic Era. Molecular biology and evolution 37, 1530-1534, https://doi.org/10.1093/molbev/msaa015 (2020). 

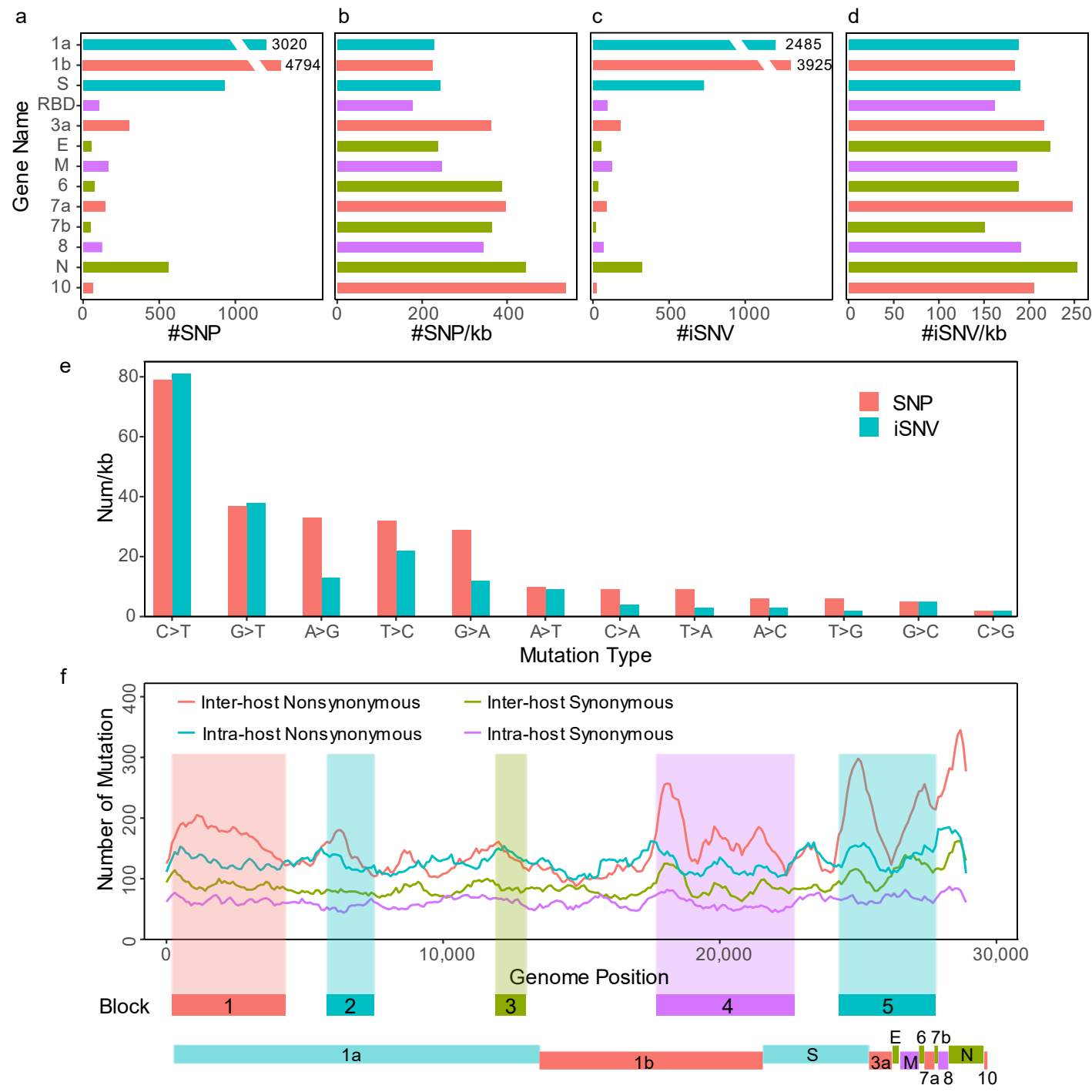

Fig. 1. Distributions of inter-host SNPs and iSNVs in the SARS-CoV-2 viral

277 a. Total number of inter-host SNPs in each ORF and RBD region. b. The number of

278 inter-host SNPs in each kb of each ORF and RBD region. c. Total number of iSNVs

279 in each ORF and RBD region. $\mathbf{d}$. The number of iSNVs for each ORF and RBD

280 region normalized by kb. e. The numbers of each SNP type normalized by kb for

281 inter-host SNPs and iSNVs. f. Distribution of nonsynonymous and synonymous

282 mutations in inter-host and intra-host SNPs. Number of mutations were calculated

283 using a sliding window with a size of $1000 \mathrm{bp}$ and a step size of $100 \mathrm{bp}$. The blocks are

284 regions having more protein gene mutations than the average level. 

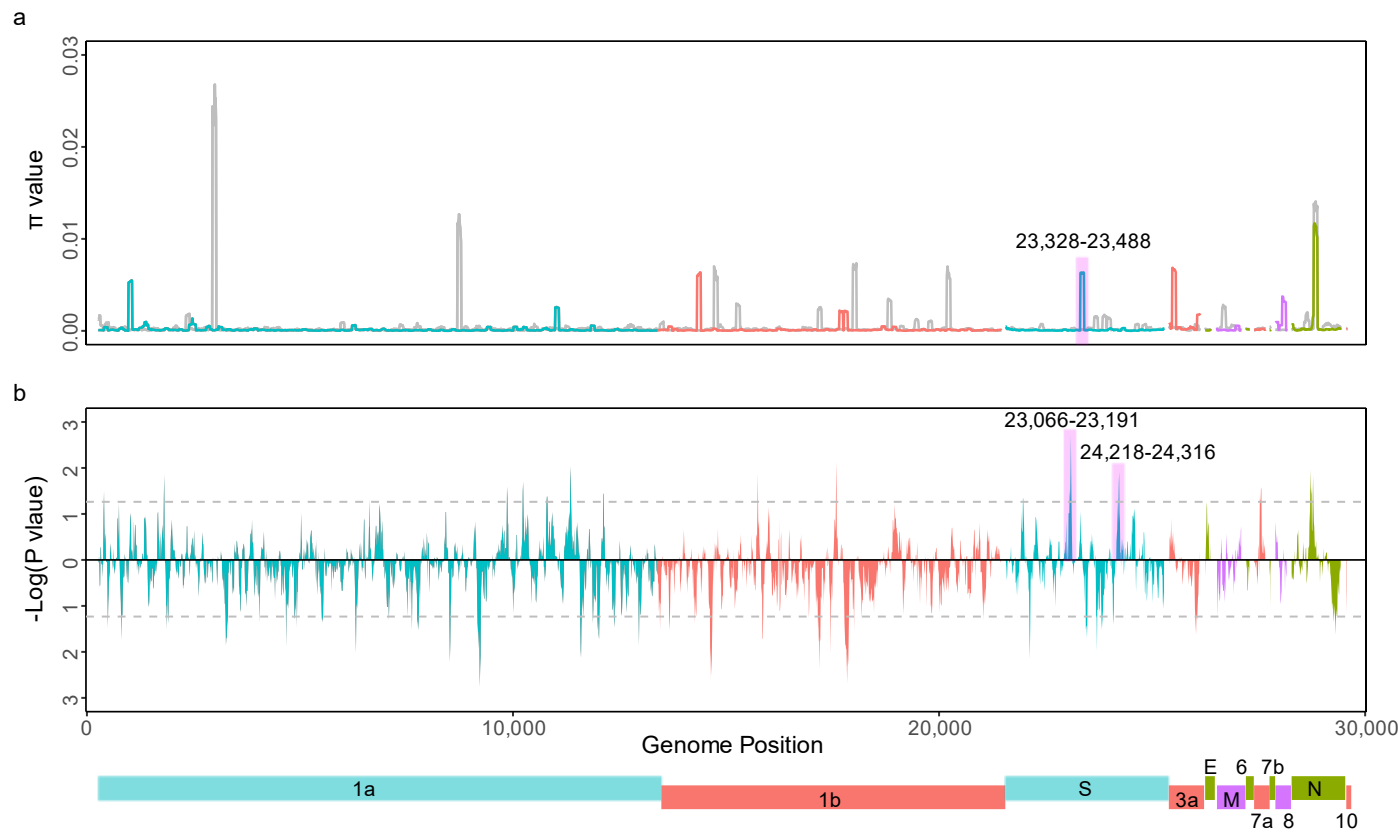

286 Fig. 2. Analysis of natural selection of the SARS-CoV-2 virus ORFs.

287 a. $\pi \mathrm{N}$ (colored lines) and $\pi \mathrm{S}$ (grey lines) were estimated for the SARS-CoV-2 virus

288 using a 30 amino acids window and 3 amino acids step. Different ORFs are

289 distinguished by different colors for $\pi \mathrm{N}$. b. The Kolmogorov-Smirnov test was

290 performed between the nonsynonymous and synonymous iSNVs mutated allele

291 frequencies (MuAFs). The upper segment indicates that the MuAF values of the

292 nonsynonymous mutation is higher than the synonymous mutation, while in the lower

293 segment the nonsynonymous mutations are lower than the synonymous mutations.

294 The dashed lines represent $\mathrm{P}<0.05$. 
a

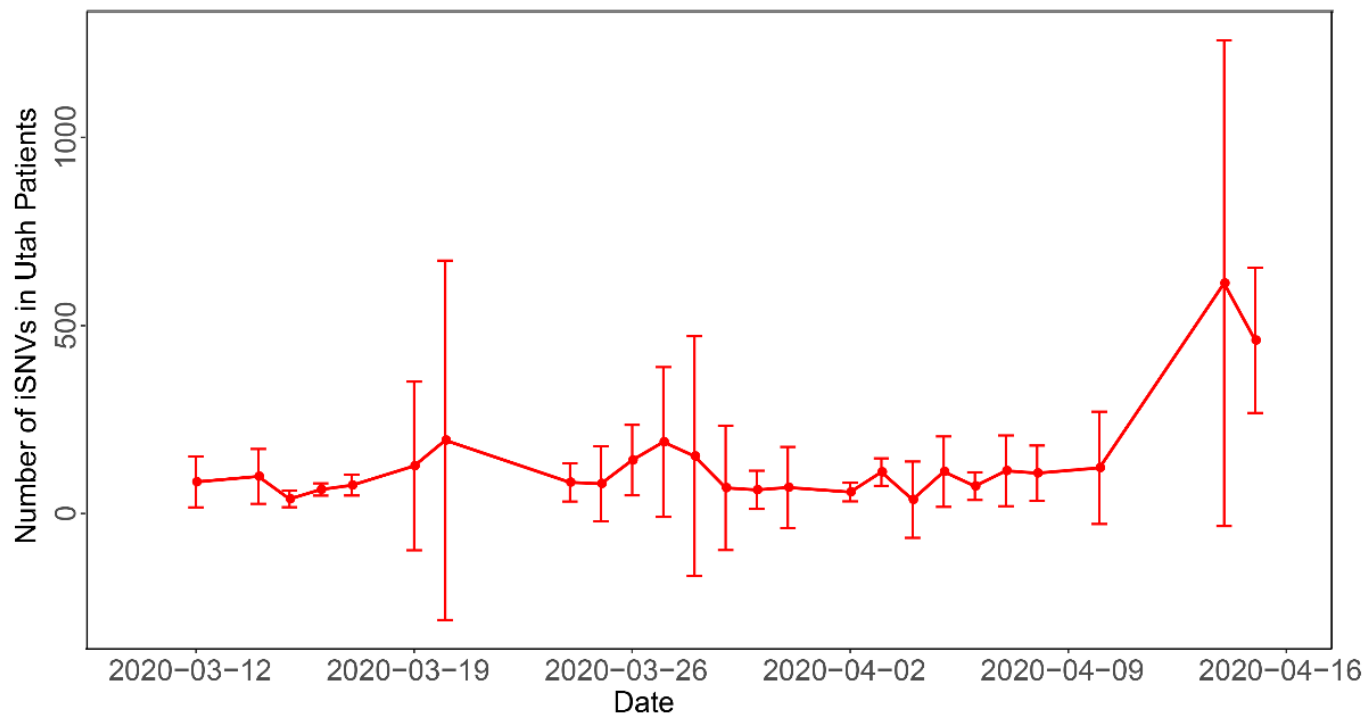

b

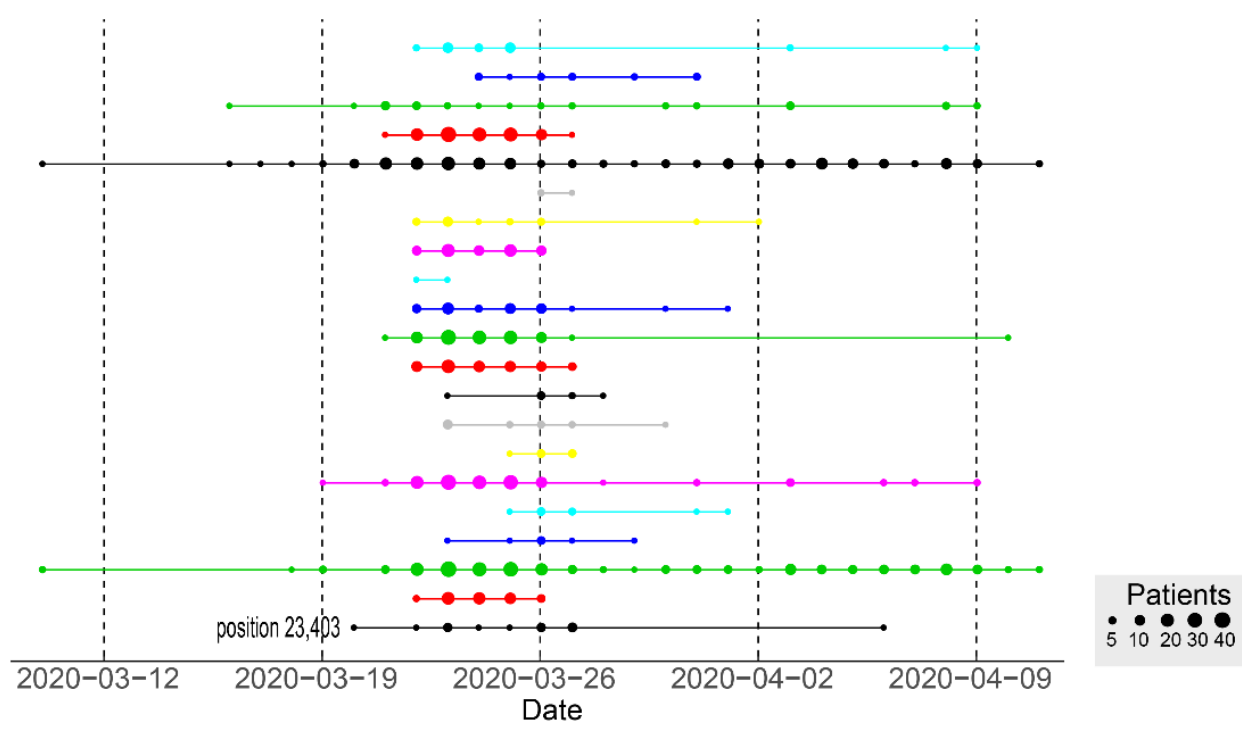

Fig. 3. Distribution and sharing of iSNVs in SARS-CoV-2 virus.

a. Time distribution of iSNVs in the Utah COVID-19 patients. Numbers indicate the number of iSNVs in all the patients of the same day. Error bars represent the $95 \%$ confidence interval. b. Evidence of human-to-human sharing in the Victoria

301 (Australia) iSNVs. SNPs shared in two or more patients are displayed as a row of 302 connected points. Each row represents a randomly selected SNP from the shared 303 iSNVs. Position 23,403 is an example of a shared fitness advantageous mutation. 
a

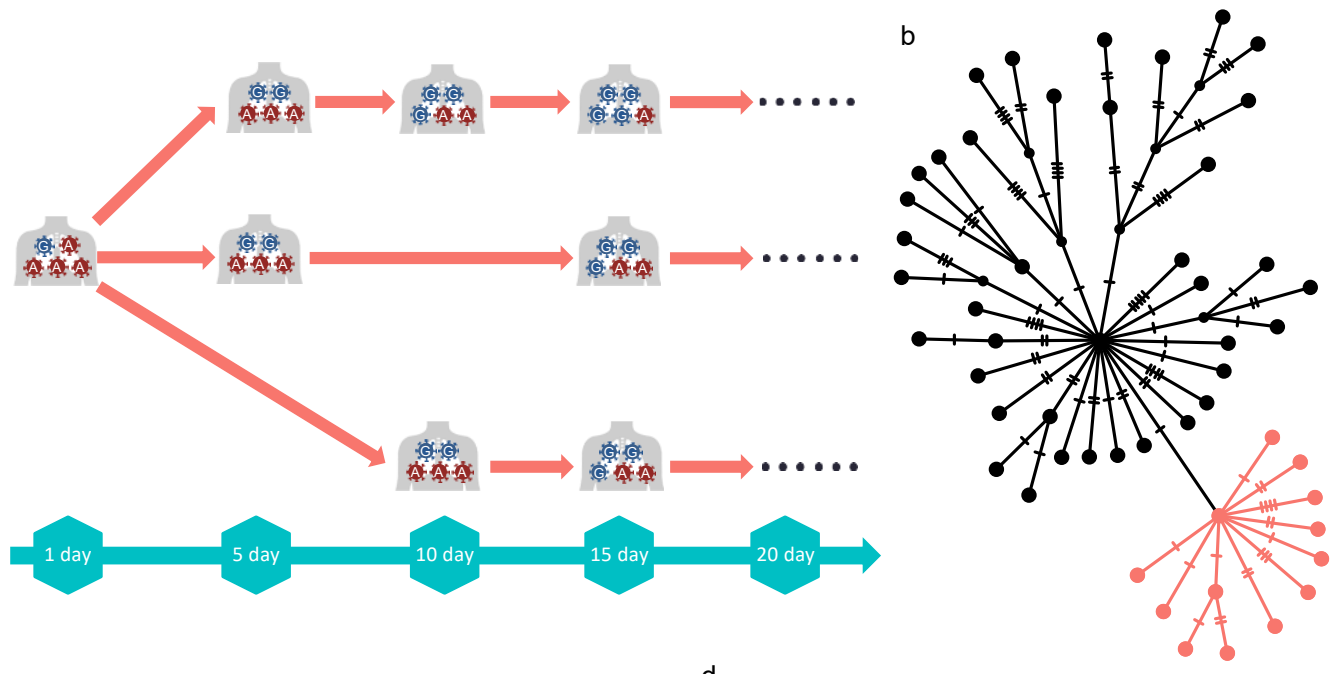

c

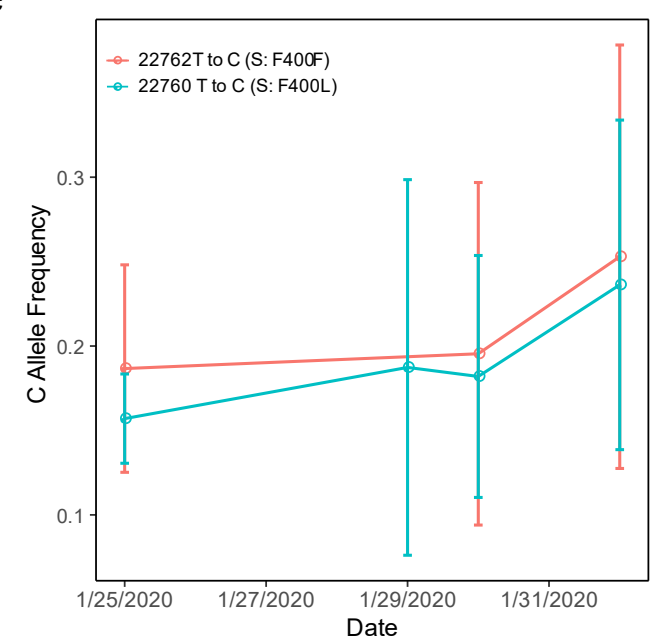

d

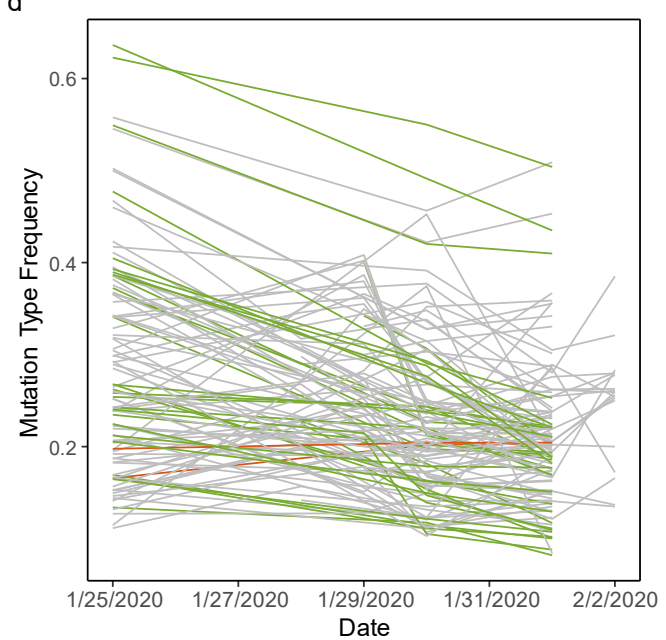

Fig. 4. Early prediction of fitness advantageous mutations for SARS-CoV-2 virus

a. Model of the human-to-human transmission of an iSNVs. G mutation is the supposed fitness advantageous mutation. b. Median-joining haplotype network constructed from the Shanghai SARS-CoV-2 genomes. Red colored dots represent a viral haplotype, which included samples used for the fitness advantageous mutation analysis. The clustered samples were assumed to be in the same transmission chain. $\mathbf{c}$.

313 Example of the early prediction of a fitness advantageous mutation. Mutation type

314 frequency was calculated as the ratio of the number of $\mathrm{C}$ reads to the sum of the 315 numbers of the $\mathrm{T}$ and $\mathrm{C}$ reads. Date was the sampling day. $\mathbf{d}$. iSNVs dynamics for 316 SARS-CoV-2 from the Shanghai patients (from a cluster marked in red in B). These iSNVs represent mutations that might be driven by neutral and purifying selection. 
318 Mutation types were inferring using the Wuhan-Hu-1 genome as the reference

319 genome. The red color represents the mutation types frequency gradually increase

320 over time. The green color indicates decrease of mutation types frequency over time.

321 Grey color represents the fluctuation of mutation types frequency. 
SARS-CoV-2 data

15,818 high-quality assembled SARS-CoV-2 sequences were acquired from the GISAID (https://www.gisaid.org/), CNGBdb (https://db.cngb.org/), GenBank

NMDC (http://nmdc.cn/nCoV) databases (on May 27, 2020). These sequences were sampled from December 2019 to May 2020 and distributed across 77 countries.

\section{Assembled sequence analysis}

All 15,817 assembled sequences were aligned against the SARS-CoV-2 reference genome (Wuhan-Hu-1, GenBank NC_045512.2) using MUSCLE version annotated to protein-coding genes and intergenic regions using ANNOVAR ${ }^{21}$.

343 data in the SRA database (accession number PRJNA627662 and PRJNA613958),

344 were aligned using MUSCLE version 3.8.31 ${ }^{11}$. Maximum likelihood phylogenetic

345 trees were constructed using IQ-TREE version 2.0.5 ${ }^{20}$. Median-joining haplotype 
346 networks were built using PopART version 1.7 (http://popart.otago.ac.nz/).

347 Phylogenetic trees and haplotype networks were used for further analyses.

\section{8 iSNVs analysis}

To obtain high-confidence iSNVs, we first trimmed low-quality bases from the

350 raw reads using Trimmomatic 0.39 with default parameters ${ }^{22}$. The passed reads were

351 mapped to the SARS-CoV-2 reference genome using BWA mem version 0.7.17 with

352 default parameters ${ }^{23}$. GATK MarkDuplicates version 4.1.3.0 was used to mark

353 duplicate reads ${ }^{24,25}$. The iSNVs were detected using bcftools mpileup version 1.10.2

354 with : mapping quality $>20$, base quality $>20$, mapping quality $>50^{26}$. iSNVs were

355 then filtered using bcftools filter version 1.10.2 with 'QUAL $<10|| \mathrm{DP}<15$ ' parameters

356 for data from India, United Kingdom, and China, and 'QUAL $<10 \| \mathrm{DP}<30$ ' for data

357 from the United States and Australia ${ }^{26}$. We used different parameters due to their

358 differences in sequencing depth. To remove errors from the sequencing and mapping

359 data, only iSNVs that were covered by at least five reads were used for further

360 analyses.

\section{Substitution rates}

Substitution rates for the Shanghai samples were assessed using the Bayesian

363 Markov chain Monte Carlo (MCMC) implemented in BEAST v1.8.4 ${ }^{15}$. We performed

364 the analysis for 100 million generations, sampling every 10,000 steps as done in a

365 previous study ${ }^{27}$.

\section{Natural selection analysis}

367 We calculated the nonsynonymous $(\pi \mathrm{N})$ and synonymous $(\pi \mathrm{S})$ nucleotide

368 diversity using SNPGeine with a script using the aligned sequences ${ }^{18}$. To intuitively

369 display the potential natural selection regions in the SARS-CoV-2 genome, we further 
370 carried out sliding window analyses (window size, 30 codons; step size, 3 codons) of

371 the $\pi \mathrm{N}$ and $\pi \mathrm{S}$.

Mutated allele frequency (MuAF) of the iSNVs were calculated using a sliding

373 window (window size, 30 codons; step size, 3 codons) with a sequencing error rate of

3740.001 and minor allele frequency of 0.001 , and other parameters as used in a previous study $^{19}$.

iSNVs dynamics analysis

To prevent a mapping bias caused by polymorphisms, the iSNVs in the SARS-

CoV-2 reference genome were replaced with ' $\mathrm{N}$ ' for each sample. We used BWA mem 0.7.17 with default parameters to re-map the reads ${ }^{23}$.

Following the haplotype network and phylogenetic tree in the Shanghai samples, we calculated the allele frequency and plotted the frequency with collection time for each lineage.

\section{Methods References}

1 Organization, W. H. WHO Coronavirus disease(COVID-19) Situation Report, https://www.who.int/emergencies/diseases/novel-coronavirus-2019/situationreports/ (2020).

2 Zhou, P. et al. A pneumonia outbreak associated with a new coronavirus of probable bat origin. Nature 579, 270-273, https://doi.org/10.1038/s41586-0202012-7 (2020).

3 Chan, J. F. et al. A familial cluster of pneumonia associated with the 2019 novel coronavirus indicating person-to-person transmission: a study of a family cluster. Lancet (London, England) 395, 514-523, https://doi.org/10.1016/s01406736(20)30154-9 (2020).

4 Wrapp, D. et al. Cryo-EM structure of the 2019-nCoV spike in the prefusion conformation. Science (New York, N.Y.) 367, 1260-1263, https://doi.org/10.1126/science.abb2507 (2020).

5 Korber, B. et al. Tracking changes in SARS-CoV-2 Spike: evidence that D614G increases infectivity of the COVID-19 virus. Cell, https://doi.org/doi: 10.1016/j.cell.2020.06.043 (2020).

6 Tang, X. et al. On the origin and continuing evolution of SARS-CoV-2. National science review, https://doi.org/doi.org/10.1093/nsr/nwaa036 (2020).

7 Park, D. J. et al. Ebola Virus Epidemiology, Transmission, and Evolution during Seven Months in Sierra Leone. Cell 161, 1516-1526, https://doi.org/10.1016/j.cell.2015.06.007 (2015). 
$8 \mathrm{Ni}$, M. et al. Intra-host dynamics of Ebola virus during 2014. Nature microbiology 1, 16151, https://doi.org/10.1038/nmicrobiol.2016.151 (2016).

9 Zhao, W. M. et al. The 2019 novel coronavirus resource. Yi chuan = Hereditas 42, 212-221, https://doi.org/10.16288/j.yczz.20-030 (2020).

$10 \mathrm{Wu}, \mathrm{F}$. et al. A new coronavirus associated with human respiratory disease in China. Nature 579, 265-269, https://doi.org/10.1038/s41586-020-2008-3 (2020).

11 Edgar, R. C. MUSCLE: multiple sequence alignment with high accuracy and high throughput. Nucleic acids research 32, 1792-1797, https://doi.org/10.1093/nar/gkh340 (2004).

12 Zhang, X. et al. Viral and host factors related to the clinical outcome of COVID19. Nature, https://doi.org/10.1038/s41586-020-2355-0 (2020).

$13 \mathrm{Wu}, \mathrm{Y}$. et al. A noncompeting pair of human neutralizing antibodies block COVID-19 virus binding to its receptor ACE2. Science (New York, N.Y.) 368, 1274-1278, https://doi.org/10.1126/science.abc2241 (2020).

14 Dai, L. et al. A Universal Design of Betacoronavirus Vaccines against COVID19, MERS, and SARS. Cell, https://doi.org/10.1016/j.cell.2020.06.035 (2020).

15 Suchard, M. A. et al. Bayesian phylogenetic and phylodynamic data integration using BEAST 1.10. Virus evolution 4, vey016, https://doi.org/10.1093/ve/vey016 (2018).

16 van Dorp, L. et al. No evidence for increased transmissibility from recurrent mutations in SARS-CoV-2. bioRxiv, https://doi.org/doi.org/10.1101/2020.05.21.108506 (2020).

17 Martin, D. P., Murrell, B., Golden, M., Khoosal, A. \& Muhire, B. RDP4: Detection and analysis of recombination patterns in virus genomes. Virus evolution 1, vev003, https://doi.org/10.1093/ve/vev003 (2015).

18 Nelson, C. W., Moncla, L. H. \& Hughes, A. L. SNPGenie: estimating evolutionary parameters to detect natural selection using pooled next-generation sequencing data. Bioinformatics (Oxford, England) 31, 3709-3711, https://doi.org/10.1093/bioinformatics/btv449 (2015).

$19 \mathrm{Li}$, Y. et al. Resequencing of 200 human exomes identifies an excess of lowfrequency non-synonymous coding variants. Nature genetics 42, 969-972, https://doi.org/10.1038/ng.680 (2010).

20 Minh, B. Q. et al. IQ-TREE 2: New Models and Efficient Methods for Phylogenetic Inference in the Genomic Era. Molecular biology and evolution 37, 1530-1534, https://doi.org/10.1093/molbev/msaa015 (2020).

21 Wang, K., Li, M. \& Hakonarson, H. ANNOVAR: functional annotation of genetic variants from high-throughput sequencing data. Nucleic acids research $\mathbf{3 8}$, e164, https://doi.org/10.1093/nar/gkq603 (2010).

22 Bolger, A. M., Lohse, M. \& Usadel, B. Trimmomatic: a flexible trimmer for Illumina sequence data. Bioinformatics (Oxford, England) 30, 2114-2120, https://doi.org/10.1093/bioinformatics/btu170 (2014).

$23 \mathrm{Li}, \mathrm{H}$. \& Durbin, R. Fast and accurate short read alignment with BurrowsWheeler transform. Bioinformatics (Oxford, England) 25, 1754-1760, https://doi.org/10.1093/bioinformatics/btp324 (2009).

24 McKenna, A. et al. The Genome Analysis Toolkit: a MapReduce framework for analyzing next-generation DNA sequencing data. Genome research 20, 1297 1303, https://doi.org/10.1101/gr.107524.110 (2010).

25 DePristo, M. A. et al. A framework for variation discovery and genotyping using next-generation DNA sequencing data. Nature genetics $\mathbf{4 3}, 491-498$, https://doi.org/10.1038/ng.806 (2011).

$26 \mathrm{Li}, \mathrm{H}$. A statistical framework for SNP calling, mutation discovery, association mapping and population genetical parameter estimation from sequencing data. 
Development Project (No. 2020YFC0847000).

Bioinformatics (Oxford, England) 27, 2987-2993,

Acknowledgments
467

\section{Corresponding author}

479 Ya-Ping Zhang, Ph.D.

480 State Key Laboratory of Genetic Resources and Evolution, Kunming Institute of

481 Zoology, Chinese Academy of Sciences, Kunming, China.

482

Email: zhangyp@mail.kiz.ac.cn

\section{Author contributions}

Y.P.Z. and Z.Y.Z. designed the project. H.L. and Z.Y.Z. performed the data analysis. Z.Y.Z. wrote the primary manuscript. Z.Y.Z., H.L., A.L., Y.D.Z. and Y.Q.W drew the figures. Y.B. provided the SARS-CoV-2 sequence alignment. M.S.P., D.M.I., H.L., J.L., X.L., D.L. and Y.P.Z. revised the manuscript.

\section{Competing interests}

The authors declare that they have no competing interests.

\section{Additional information}

This work was supported by the Chinese Academy of Sciences, National Nature 


\section{$484 \quad$ Extended data table legends}

485 Extended Data Table 1. SNPs identified in the assembled SARS-CoV-2 sequences.

486 Extended Data Table 2. Acknowledgement of the sharing of SARS-CoV-2 raw

487 genome sequences in NCBI SRA database used in this study.

488 Extended Data Table 3. Annotations of the SNPs identified from the raw reads

489 generated from next generation sequencing of SARS-CoV-2 samples.

490 Extended Data Table 4. Estimates of the substitution rate for the SARS-CoV-2

491 genome and the regions with high numbers of mutations in protein genes. HPD: 95\%

492 highest posterior density interval.

493 Extended Data Table 5. Mean nonsynonymous and synonymous nucleotide diversity 494 of each ORF. 


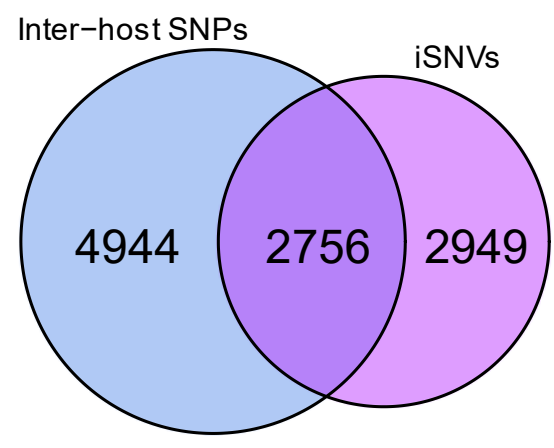

496 Extended Data Fig.1. Overlapping of SNPs identified in the inter-host and the

497 intra-host.

498 


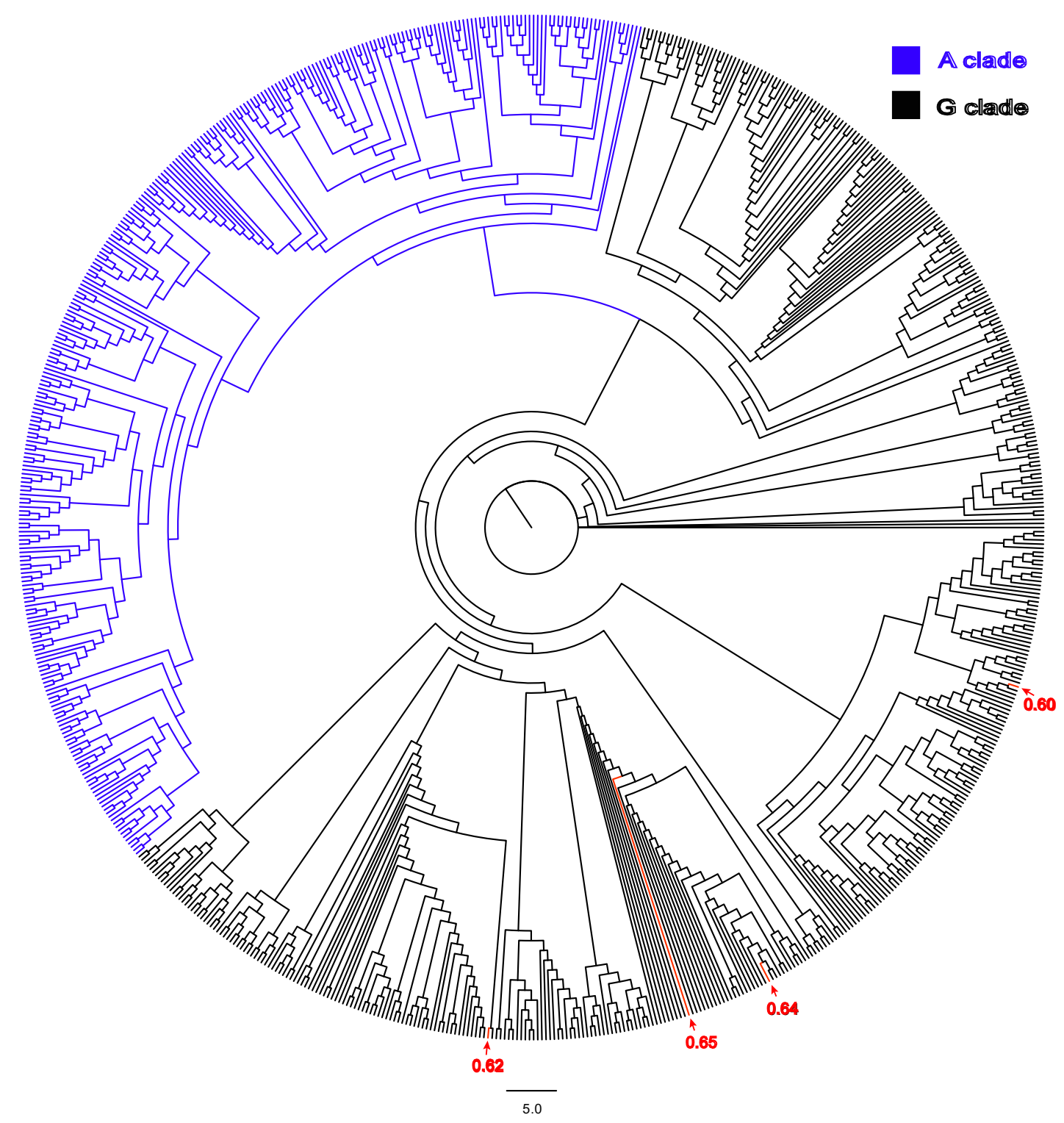
SNP at position 23,403 .

502 The frequency of the $\mathrm{G}$ mutation type was calculated as the ratio of number of $\mathrm{G}$

503 reads to the sum of numbers of A and G reads. Samples related to the intermediate

504 frequency are marked in red. 


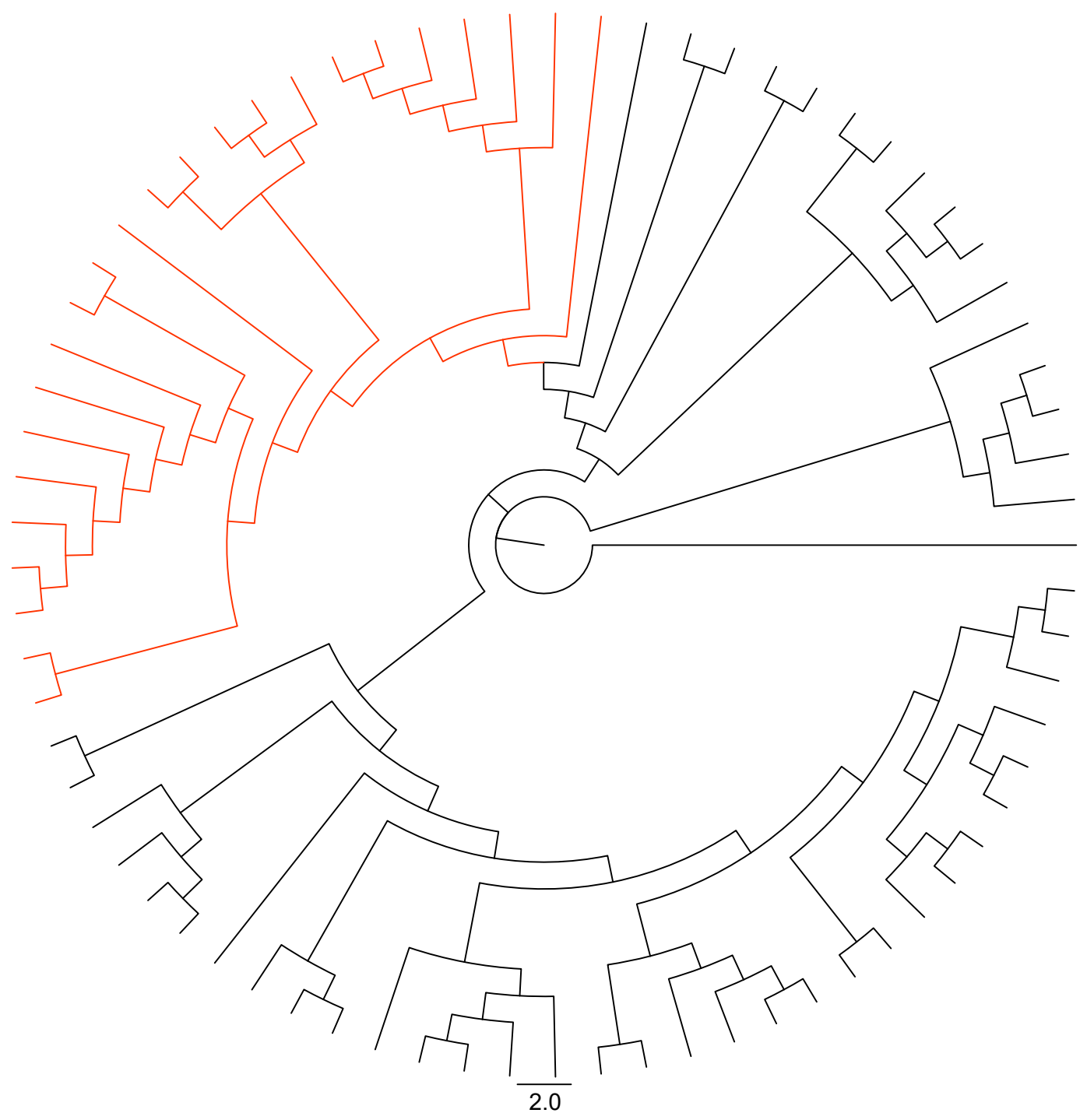

Extended Data Fig.3. Phylogenetic placement of the Figure 3B viral haplotype (marked in red).

509 Clustered samples were assumed to be in the same transmission chain. 
a

512

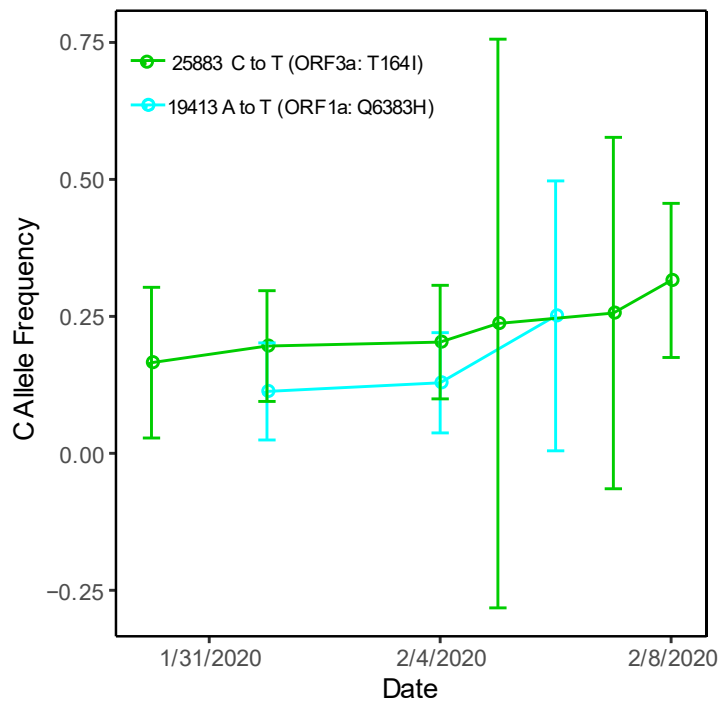

b

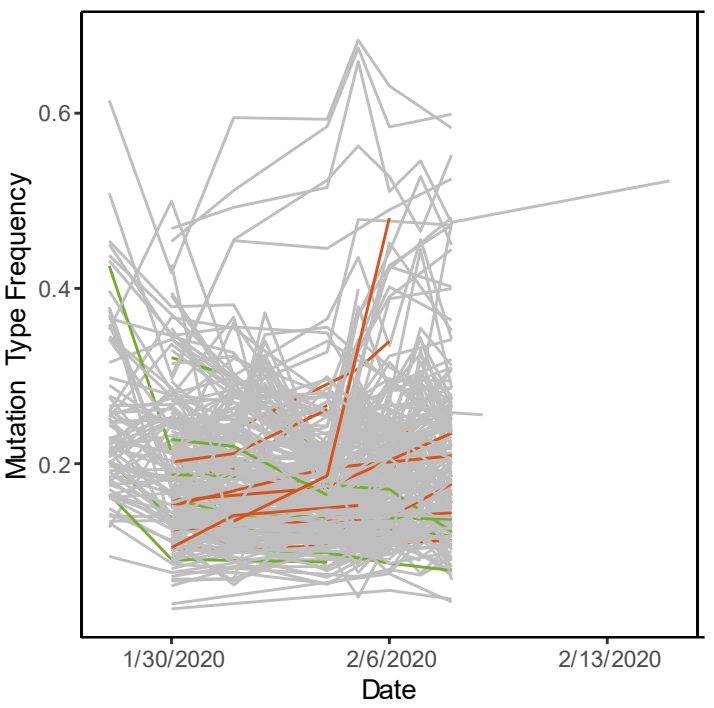

513 Extended Data Fig.4. Example of other candidate fitness advantageous

514 mutations.

515 a. Two candidate fitness advantageous mutations identified in the Shanghai patients.

516 Mutation types were inferring using the Wuhan-Hu-1 genome as the reference

517 genome. Mutation type frequency was calculated as the ratio of number of mutation

518 type reads to the sum of numbers of wild- and mutation-type reads. Date was the

519 sampling day. b. iSNVs dynamics for the Shanghai patients (from the cluster labeled

520 in black in Fig. 4B). These iSNVs may be driven by neutral and purifying selection. 